\title{
Myocardial remodeling with aortic stenosis and after aortic valve replacement: Mechanisms and future prognostic implications
}

\author{
William M. Yarbrough, MD, ${ }^{\mathrm{a}}$ Rupak Mukherjee, $\mathrm{PhD},{ }^{\mathrm{a}} \mathrm{John} \mathrm{S}$. Ikonomidis, $\mathrm{MD}, \mathrm{PhD},{ }^{\mathrm{a}}$ \\ Michael R. Zile, MD, ${ }^{\text {,c }}$ and Francis G. Spinale, MD, $\mathrm{PhD}^{\mathrm{a}, \mathrm{c}}$
}

Aortic valve stenosis is a common cause of left ventricular pressure overload, a pathologic process that elicits myocyte hypertrophy and alterations in extracellular matrix composition, both of which contribute to increases in left ventricular stiffness. However, clinical and animal studies suggest that increased myocardial extracellular matrix fibrillar collagen content occurs later in the time course of left ventricular pressure overload at a time coincident with severe abnormalities in diastolic function followed by the development of symptomatic heart failure. Aortic valve replacement remains the most effective treatment for elimination of chronic pressure overload secondary to aortic stenosis but has traditionally been recommended only after the onset of clinical symptoms. Long-term follow-up of patients with symptomatic aortic stenosis after aortic valve replacement suggests that valve replacement may not result in complete reversal of the maladaptive changes that occur within the myocardial extracellular matrix secondary to the pressure overload state. To the contrary, residual left ventricular extracellular matrix abnormalities such as these are likely responsible for persistent abnormalities in diastolic function and increased morbidity and mortality after aortic valve replacement. Defining the mechanisms and pathways responsible for regulating the myocardial extracellular matrix during the natural history of aortic stenosis may provide a means by which to detect crucial structural milestones and thereby permit more precise identification of the development of maladaptive left ventricular remodeling. (J Thorac Cardiovasc Surg 2012;143:656-64)

Aortic stenosis (AS) is a common disease in which failure of the aortic valve to completely open imposes an abnormally high-pressure load on the left ventricle (LV). Irrespective of the cause of AS, the ensuing pressure overload results in the manifestation of 2 distinct but overlapping processes. $^{1-3}$ The first process is characterized by concentric left ventricular hypertrophy $(\mathrm{LVH})$, and, as demonstrated by the Law of Laplace, the increased wall thickness and mass act to limit the increase in wall stress created by the ASinduced pressure overload state. ${ }^{2,4}$ The second process occurs within the myocardial extracellular matrix (ECM) and leads to progressive myocardial fibrosis, reduced ventricular compliance, and impairments in diastolic filling (ie, diastolic dysfunction). ${ }^{5-10}$ It is this second phase of progressive LV myocardial fibrosis that contributes to the progression of LV diastolic dysfunction and eventually to the presentation of the signs and symptoms of heart failure. ${ }^{6,10}$

Aortic valve replacement (AVR) remains the single most effective intervention for long-standing elimination of

\footnotetext{
From the Divisions of Cardiothoracic Surgery ${ }^{\mathrm{a}}$ and Cardiology, ${ }^{\mathrm{b}}$ Department of Medicine, Medical University of South Carolina, Charleston, SC; and Ralph H. Johnson Veterans' Association Medical Center, ${ }^{\mathrm{c}}$ Charleston, SC.

This work was supported by the Third Edward D. Churchill Research Scholarship (American Association for Thoracic Surgery), a National Institutes of Health Supplement award (HL 57952), and the Veterans' Affairs Health Administration.

Disclosures: Authors have nothing to disclose with regard to commercial support.

Received for publication Dec 17, 2010; revisions received March 11, 2011; accepted for publication April 11, 2011; available ahead of print July 15, 2011.

Address for reprints: Francis G. Spinale, MD, PhD, Cardiothoracic Surgery, Strom Thurmond Research Center, 114 Doughty St, Suite 625, Charleston, SC 29425 (E-mail: wilburnm@musc.edu)

0022-5223/\$0.00

Published by Elsevier Inc. on behalf of The American Association for Thoracic Surgery

doi:10.1016/j.jtcvs.2011.04.044
}

pressure overload in patients with AS. The current guidelines for AVR in patients with AS include those with severe AS and the presence of symptoms such as heart failure. ${ }^{11}$ In the absence of precluding factors, the presence of symptoms with AS mandates consideration for AVR to avoid a $25 \%$ yearly mortality rate associated with disease of this extent. ${ }^{12}$ However, there are several lines of evidence to suggest that this paradigm for timing of AVR could be improved. First, forestalling AVR until heart failure symptoms manifest implies that the development of the decompensated maladaptive phase has already occurred. As a result, significant and deleterious structural LV myocardial remodeling has likely already ensued along with the attendant changes in LV diastolic function. ${ }^{6}$ Although imaging and functional studies such as echocardiography can quantify indices of LV diastolic function (eg, filling rates and relaxation times), abnormalities in these indices will only become detectable once the structural changes within the myocardium and in particular the myocardial ECM have already become manifest. Thus, once physiologically and subsequently clinically significant LV diastolic dysfunction has occurred with AS, significant and irreversible changes within the myocardial ECM have been established. Second, significant clinical and experimental evidence exists to suggest that when AVR is performed only after the development of LV diastolic dysfunction and heart failure symptoms, the LV myocardial remodeling process that ensued from long-standing AS may not be readily reversible. ${ }^{5-7,13}$ Myocardial fibrosis secondary to long-standing and progressive AS may persist for years after $\mathrm{AVR}^{6}$ and can contribute to the persistence of postoperative heart failure symptoms. ${ }^{13,14}$ Therefore, although the 


$$
\begin{aligned}
& \text { Abbreviations and Acronyms } \\
& \mathrm{ARB}=\text { angiotensin receptor blockade } \\
& \text { AS = aortic stenosis } \\
& \text { AVR = aortic valve replacement } \\
& \mathrm{BNP}=\mathrm{B} \text {-type natriuretic peptide } \\
& \mathrm{CT}=\text { cardiotrophin } \\
& \mathrm{ECM}=\text { extracellular matrix } \\
& \mathrm{LV}=\text { left ventricle, left ventricular } \\
& \text { LVH = left ventricular hypertrophy } \\
& \text { MMP = matrix metalloproteinase } \\
& \text { mRNA }=\text { messenger RNA } \\
& \mathrm{TGF}=\text { transforming growth factor } \\
& \text { TIMP }=\text { tissue inhibitor of matrix } \\
& \text { metalloproteinase }
\end{aligned}
$$

use of current guidelines to determine appropriate timing of AVR has yielded reasonable surgical outcomes, this approach may result in the incomplete reversal of the deleterious changes present within the LV myocardial matrix of patients with symptomatic AS.

To improve the timing for AVR, it is essential to identify the mechanistic underpinnings that contribute to the development of maladaptive LV remodeling. Because increased myocardial fibrosis and myocardial stiffness represent hallmarks of maladaptive $\mathrm{LV}$ remodeling with $\mathrm{AS},{ }^{10,15}$ the determinants of ECM remodeling likely play an instrumental role. Although elucidation of molecular and biochemical pathways that contribute to myocardial ECM remodeling, fibrosis, and LV dysfunction will provide clinically relevant prognostic information, translation of these basic ECM pathways into diagnostic and prognostic applications that can be used to direct appropriate timing for AVR within the context of AS is necessary. Accordingly, the purpose of this review is 2-fold. The first goal is to examine the results obtained from clinical and relevant preclinical studies of AS and AVR with respect to adverse LV ECM remodeling and the attendant effects on LV form and function. Although significant structural and ECM changes occur within the aortic valve itself with AS, the focus of this review is to examine the consequences of the pressure overload with AS, as it applies to the myocardium. The specific focus of this goal will be to examine the temporal and structural LV remodeling that exists in the ECM compartments during the development and progression of AS-induced LVH and heart failure and after relief from this pressure overload state with AVR. We will attempt to coalesce the findings generated in these studies to provide an updated hypothesis of the molecular pathways that regulate myocardial ECM remodeling with AS and after AVR. The second goal of this review is to examine molecular pathways that contribute to myocardial ECM structure and composition and identify those pathways that may yield relevant biomarker signatures with respect to the natural history of AS. This goal will allow development of methods to improve the timing of AVR.

\section{CLINICAL INVESTIGATIONS OF AORTIC STENOSIS}

Although increased myocyte size is one critical feature of pressure overload-induced LV remodeling, the development of abnormalities within the ECM represents a maladaptive structural milestone in the progression of decompensated heart failure..$^{5-7,16-18}$ Changes in ECM composition and structure and accumulation of ECM components, such as increased fibrillar collagen content, have been shown to impair LV diastolic filling and decrease LV distensibility in hypertensive heart disease $^{16,17}$ and clinical AS. ${ }^{7,10,13,19,20}$ These pressure overload-induced changes in ECM structure and composition play a mechanistic role in the progression of heart failure. There are several clinical studies that support this conclusion and clearly relate myocardial ECM content to LV diastolic mechanics during the progression of ASinduced pressure overload and during the regression of AS-induced pressure overload after AVR. These investigations are briefly described next.

A specific set of cellular and extracellular events occur during the development of AS and after AVR. Investigators from the University Hospital, Zurich, Switzerland, provided much of what is known regarding the effects of clinical aortic valve stenosis on myocardial structure and function before and after AVR. ${ }^{5-8}$ Patients with AS demonstrated significantly increased LV myocardial interstitial fibrosis compared with control patients. $^{6-8}$ Moreover, LV relaxation, filling, and stiffness were abnormal in patients with AS. ${ }^{6}$ However, there were no changes in LV systolic performance in patients with AS. ${ }^{5,6}$ Thus, these investigations are important in that they linked changes within the LV extracellular compartment with the clinical syndrome of pressure overload-induced diastolic dysfunction and heart failure. These same investigators examined the effects of AVR on components of the myocardial ECM. $^{6}$ Specifically, AVR was associated with changes in interstitial fibrosis at both early (22 \pm 8 months) and late (81 \pm 24 months) time points compared with preoperative values. Interstitial fibrosis remained elevated over control values late after AVR and failed to normalize (Figure 1). Whether the persistent LV structural abnormalities noted by these investigators achieve clinical significance remains a matter of debate; however, it is noteworthy that these colleagues demonstrated that approximately $20 \%$ of patients with AS exhibited some degree of exercise intolerance 5 to 10 years after AVR. ${ }^{14}$ A more recent clinical investigation conducted by Weidemann and associates ${ }^{15}$ lends credence to the assertion made by these initial investigators 

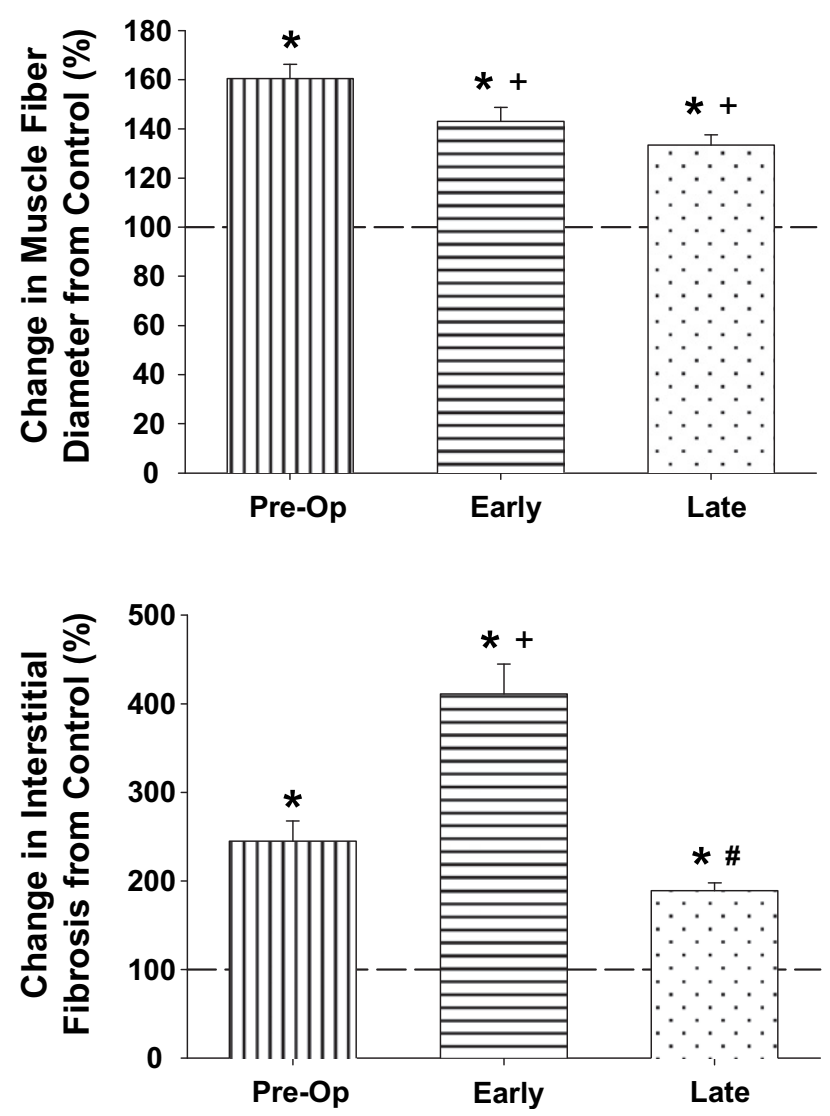

FIGURE 1. Villari and colleagues ${ }^{6}$ demonstrated that regression of AS-induced cellular and extracellular abnormalities occurs in a timedependent manner after AVR. Moreover, elimination of the pressure overload state failed to achieve normalization of myocyte diameter and interstitial fibrosis. Expressed as a percentage change from control values, AVR was associated with significant reductions in muscle fiber diameter at both early ( $22 \pm 8$ months) and late $(81 \pm 24$ months $)$ time points compared with preoperative values; however, muscle fiber diameter remained significantly elevated over control values late after AVR. Values for interstitial fibrosis increased early after AVR compared with preoperative values. This increase was likely "relative" and a result of a concomitant early reduction in myocyte mass as opposed to an increase in collagen synthesis after AVR. Values for interstitial fibrosis remained significantly elevated above those observed in the control group late after AVR. Data are presented as the mean and the standard error of the mean and were modified from Villari and colleagues ${ }^{6}(* P<.05$ vs control, $+P<.05$ vs preoperative, $\# P<.05$ vs early).

that AVR may fail to completely reverse AS-induced myocardial fibrosis. In their prospective follow-up study, Weidemann and colleagues determined that the degree of myocardial fibrosis (biopsy taken at the time of operative intervention) correlated more closely with the preoperative New York Heart Association class than LV ejection fraction or aortic valve area in patients undergoing valve replacement for AS. Moreover, they determined that AVR provided less functional improvement in patients with AS and severe fibrosis compared with those patients with lesser degrees of
MYOCARDIAL FIBROSIS - Histopathology (\%)

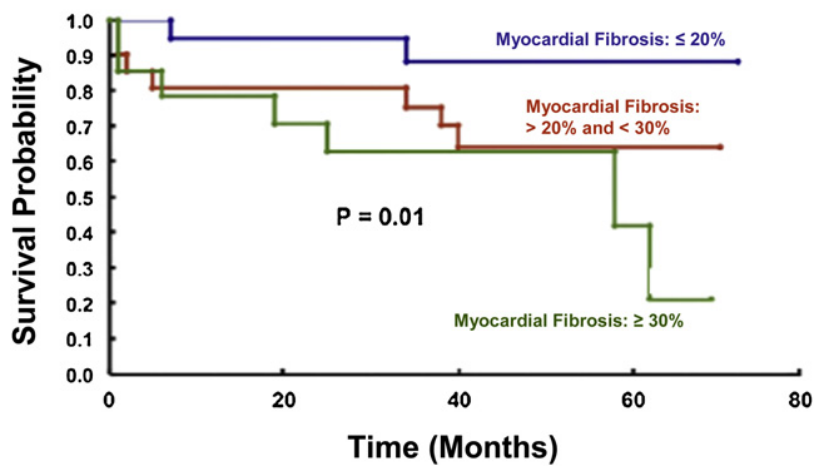

FIGURE 2. Increasing degrees of myocardial fibrosis secondary to aortic valve disease (as assessed histologically) are associated with progressive reductions in survival in patients treated with AVR using traditional criteria for intervention (ie, symptom onset). Modified from Azevedo and colleagues. $^{13}$

fibrosis. Although their period of follow-up ( 9 months) was substantially shorter than that described in the preceding clinical investigations, it is noteworthy that these authors used magnetic resonance imaging studies to demonstrate that AVR failed to reduce fibrosis in the majority of patients with severe fibrosis at baseline. Azevedo and colleagues ${ }^{13}$ similarly used histopathology and magnetic resonance imaging to assess AS-induced myocardial fibrosis. They demonstrated that greater degrees of myocardial fibrosis were associated with decreased survival after AVR (Figure 2). Taken together, the results of these clinical investigations suggest that consideration for valve replacement before the onset of adverse myocardial ECM sequelae may represent a worthwhile goal in patients with severe but asymptomatic AS. Moreover, consideration for such a deviation from current practice is only strengthened by the realization that isolated AVR has become a relatively safe operation associated with an overall mortality rate approaching $3 \%$, a value far less than that observed during the conduction of the landmark clinical investigations described above..$^{5-7}$

It is noteworthy that the degree of observed myocardial fibrosis secondary to AS appears to differ among patients with preserved LV systolic function and those with LV systolic dysfunction. As alluded to above, Weidemann and colleagues ${ }^{15}$ assessed LV myocardial fibrosis in patients with severe AS and preserved LV ejection fraction. The degree of fibrosis observed among these patients varied widely from minimal to severe. However, it is likely that this disparity diminishes as LV systolic function deteriorates as a result of long-standing LV pressure overload. Investigations examining the effects of long-standing pressure overload secondary to hypertensive heart disease ${ }^{17}$ and $\mathrm{AS}^{10}$ suggest that fibrosis is increased in patients with reduced LV ejection fraction. For example, Querejeta and colleagues ${ }^{17}$ demonstrated that collagen deposition was greater in patients 
with hypertension and heart failure compared with patients with hypertension alone. Hein and colleagues ${ }^{10}$ examined the transition from compensated LVH to heart failure in patients with AS and demonstrated that the fibrotic response was greater in patients with reduced LV ejection fractions. Thus, although the degree of observed LV myocardial fibrosis may vary among patients with preserved LV systolic function and severe pressure overload, it seems that marked fibrosis tends to accompany patients with LV systolic dysfunction and pressure overload. Such a realization highlights that fact that progressive myocardial fibrosis contributes to the signs and symptoms of heart failure secondary to long-standing pressure overload.

On assessment of the clinical investigations included in this review, it would seem reasonable to assume that as the aortic valve area decreases or the transvalvular mean gradient increases that LV fibrosis would increase in a linear fashion. However, this does not seem to be the case. The majority of the investigations considered above did not include individual subject values for these parameters, and this renders efforts to assess correlation problematic. In an effort to address this issue, we analyzed individual subject data points provided in a clinical investigation conducted by Peterson and colleagues. ${ }^{9}$ These investigators assessed patients with moderate to severe AS, and they included individual subject values for $\mathrm{LV}$ ejection fraction, aortic valve area, transvalvular mean gradient, and myocardial stiffness. Although it is true that myocardial stiffness can be altered by both cellular and extracellular characteristics, it seems reasonable to consider myocardial stiffness as a surrogate for myocardial fibrosis. Accordingly, on the basis of information provided by Peterson and colleagues, we performed linear regression analyses to determine whether relationships existed among LV ejection fraction, aortic valve area, and transvalvular mean gradient with myocardial stiffness. There were no significant relationships between these parameters and myocardial stiffness. These findings are in keeping with those of past investigations. For example, Krayenbuehl and colleagues ${ }^{7}$ found no significant correlation between $L V$ ejection fraction and percentage of interstitial fibrosis (as assessed by histology) in patients with severe AS. However, it should be noted that these studies included patients with preserved LV systolic function as assessed by LV ejection fraction. It is certainly possible that had patients with decompensated heart failure been included, a correlation might have been observed. Therefore, because there seems to be no clear relationship between fibrosis and LV ejection fraction, aortic valve area, and transvalvular mean gradient in patients with AS, a new effort should be made to identify an alternative strategy by which to monitor patients with AS and to guide timeliness of surgical intervention. As evidenced by the data recently reported by Azevedo and colleagues, ${ }^{13}$ further improvements can likely be made in reducing mortality and postoperative outcomes in patients undergoing AVR for AS, and it is likely that the key to accomplishing this task lies within the myocardial ECM.

\section{MODELS OF CHRONIC PRESSURE OVERLOAD}

Short of carrying out serial myocardial biopsies in clinical settings, it is difficult to assess the effects of chronic LV pressure overload on the LV myocardial ECM. It is fortunate that preclinical investigations describing the effects of chronic pressure overload on myocardial structure and function in large animals do exist, ${ }^{21-24}$ although they are relatively few in number in comparison with small animal pressure overload constructs. Because gradual increases in the pressure overload state can be carried out more easily in large animals, these models provide an opportunity to closely recapitulate the clinical context of AS. For example, Moorjani and collaborators ${ }^{21}$ banded sheep with variable constrictors and gradually increased the degree of stenosis to achieve peak terminal gradients approximating $80 \mathrm{~mm} \mathrm{Hg}$. LV mass doubled within 1 month after the onset of progressive and sustained aortic constriction. Investigators in Leipzig, Germany, are also credited with establishing a chronic ovine model of chronic pressure overload.22 Supracoronary banding was carried out in growing sheep to mimic compensated LV pressure overload, and LV morphologic assessments were carried out approximately 8 months later. These assessments revealed significant increases in myocardial fiber index, LV mass index, and wall thickness compared with baseline and control values. The importance of these experimental models cannot be overstated. Because of the models' ability to recapitulate the common clinical scenario of compensated AS, an opportunity exists in which changes in myocardial ECM properties secondary to chronic pressure overload can be serially assessed. Thus, it seems likely that similar model constructs can be used to more closely identify critical ECM events that are responsible for igniting maladaptive $L V$ remodeling events in the setting of chronic pressure overload. Taken together, the results of these experimental and clinical studies suggest that some degree of plasticity exists in the progression of morphologic changes that occur secondary to pressure overload. However, the time point at which plasticity is lost within the myocardial ECM, and reversibility of maladaptive myocardial changes is no longer possible, remains elusive. Identification of specific surrogate markers within the peripheral circulation that are reflective of adverse ECM events may provide an avenue for the identification of just such a transition point.

\section{LEFT VENTRICULAR PRESSURE OVERLOAD AND IDENTIFICATION OF ABNORMAL EXTRACELLULAR MATRIX PATHWAYS}

Biomarkers are measurable serum proteins that are associated with various disease states, including AS. For 
TABLE 1. Potential biomarkers for tracking aortic stenosis

\begin{tabular}{|c|c|}
\hline Osteopontin & $\begin{array}{l}\text { - Glycophosphoprotein that stimulates differentiation of myofibroblasts into osteoblasts. } \\
\text { - Serum levels are increased in AS. }\end{array}$ \\
\hline C-reactive protein & $\begin{array}{l}\text { - Acute-phase reactant that is increased in inflammatory states (ie, AS). } \\
\text { - Serum levels can be used to predict symptom severity associated with AS. }{ }^{26}\end{array}$ \\
\hline BNP & $\begin{array}{l}\text { - Levels of this endogenous cardiac hormone correlate with New York Heart Association symptom class and are elevated in } \\
\text { patients with AS-induced pressure overload. }\end{array}$ \\
\hline TGF- $\beta$ & - Cytokine that induces fibroblast activation and collagen production/fibrosis in response to pressure overload..$^{30,33}$ \\
\hline $\mathrm{CT}-1$ & - Cytokine derived from cardiac fibroblasts ${ }^{34}$ that induces myocyte hypertrophy ${ }^{35}$ and stimulates fibroblast growth. ${ }^{36}$ \\
\hline $\begin{array}{l}\text { Collagen-derived peptides } \\
\text { (PIP, PIIIP, CITP) }\end{array}$ & $\begin{array}{l}\text { - Because serum values of collagen-derived peptides reflect collagen synthesis and degradation, their measured abundances } \\
\text { may be used to assess the severity and progression of pressure overload states. }{ }^{43}\end{array}$ \\
\hline MMPs & $\begin{array}{l}\text { - Zinc-dependent proteolytic enzymes that degrade the myocardial ECM. } \\
\text { - Influence bioactive signaling pathways by processing cytokines/cytokine receptors. }{ }^{46,57}\end{array}$ \\
\hline TIMPs & $\begin{array}{l}\text { - Endogenous proteins that bind to MMPs and regulate their proteolytic activity. } \\
\text { - TIMP-1 and } 2 \text { have been most extensively investigated in the setting of heart failure. }{ }^{46,47,58}\end{array}$ \\
\hline
\end{tabular}

$A S$, Aortic stenosis; $B N P$, B-type natriuretic peptide; $T G F$, transforming growth factor; $C T$, cardiotrophin; $E C M$, extracellular matrix; $C I T P$, collagen I telopeptide; PIP, procollagen type I carboxy-terminal propeptide; PIIIP, procollagen type III amino-terminal propeptide; MMP, matrix metalloproteinase; TIMP, tissue inhibitor of matrix metalloproteinase.

biomarkers to be considered ideal, they should be easily measurable and reproducible among separate laboratories, and should provide clinicians with at least some information regarding disease prognosis and treatment efficacy, or lack thereof. The past decade is marked by a host of clinical and experimental investigations aimed at identifying biomarkers geared toward identification of patients with clinically significant AS who may benefit from AVR, and these include osteopontin, C-reactive protein, and B-type natriuretic peptide (BNP). ${ }^{25-28}$ For example, clinical studies performed by Gerber and associates ${ }^{25}$ provided evidence that BNP may be used to monitor and reflect symptom onset in patients with AS. Their studies demonstrated that yearly increases in BNP values were greater in patients with AS who developed symptoms compared with those who did not. Furthermore, these investigators established that the typical parameters used to direct therapeutic intervention for patients with AS, such as aortic valve area, transvalvular peak velocity, and LV ejection fraction, were less reliable than increases in BNP in predicting symptom onset. Neverdal and colleagues ${ }^{29}$ demonstrated that plasma BNP values decreased after AVR and that the observed decrease correlated with regression in the LV mass index. However, it should be noted that although biomarkers such as these may be used to assess the severity of clinical AS, detection of robust serum values likely corresponds to the latter stages of the disease process. In other words, marked elevations of C-reactive protein and BNP in the setting of AS likely occur only after the transition from adaptive to maladaptive LV remodeling. Accordingly, it is imperative that biomarkers with greater sensitivity for identification of just such a transition be identified to more accurately time AVR for patients with AS. Identification of such a critical time point would be more likely to facilitate complete regression of adverse myocardial cellular and extracellular changes that ensue secondary to long-standing pressure overload. Because myocyte hypertrophy represents an early and relatively reversible event in the progression of pressure overload induced myocardial remodeling, it seems appropriate to focus attention on the identification of biomarkers that are more closely associated with remodeling of the myocardial ECM, that is, those pertaining to fibrosis (Table 1). Such biomarkers include, but are not limited to, transforming growth factor (TGF)- $\beta$, cardiotrophin (CT)-1, collagenderived peptides (carboxy-terminal propeptide of procollagen I and carboxy-terminal telopeptide of procollagen I), matrix metalloproteinases (MMPs), and tissue inhibitors of the matrix metalloproteinases (TIMPs).

\section{TRANSFORMING GROWTH FACTOR- $\beta 1$}

TGF- $\beta$ is a multifunctional cytokine that is responsible for inducing collagen production and known to regulate cellular proliferation and differentiation in both normal homeostatic pathways and numerous disease states. ${ }^{30,31}$ Past experimental investigations have implicated this protein as a critical entity in the orchestration of deleterious changes within the myocardial cellular and extracellular compartments in the setting of pressure overload. ${ }^{32,33}$ For example, Kuwahara and colleagues applied a TGF$\beta$ blocking antibody to a rodent model of pressure overload and bolstered proof of concept evidence that TGF- $\beta$ plays a crucial role in carrying out the elaboration of ECM components in the setting of pressure overload. ${ }^{33}$ These investigators observed inhibition of fibroblast activation, collagen messenger RNA (mRNA) induction, and fibrosis in pressure-overloaded rodents treated with the neutralizing antibody. In an investigation that highlighted TGF- $\beta$ as a potential biomarker, Villar and associates demonstrated that plasma levels of TGF- $\beta$ were significantly increased in patients with $\mathrm{AS}$ and in mice subjected to transaortic constriction compared with controls. ${ }^{32}$ Moreover, plasma TGF- $\beta$ levels positively correlated with 
transvalvular mean gradients and representative parameters for LV mass in patients with AS before and after AVR. A positive correlation between TGF- $\beta$ and genes encoding collagen I and III was also identified.

\section{CARDIOTROPHIN-1}

Cardiac CT-1 is a cytokine that is primarily derived from cardiac fibroblasts ${ }^{34}$ and that has been shown to induce myocyte hypertrophy ${ }^{35}$ and stimulate fibroblast growth. ${ }^{36} \mathrm{~A}$ recent clinical investigation by López and colleagues ${ }^{37}$ demonstrated that CT-1 could be used as a biomarker to assess the severity and progression of LV pressure overload secondary to hypertensive heart disease. Specifically, these investigators studied patients with hypertension and no cardiac disease, patients with hypertension and $\mathrm{LVH}$, and patients with hypertension, $\mathrm{LVH}$, and signs of chronic heart failure. Plasma CT-1 levels were assessed and found to increase progressively with increasing stages of cardiac dysfunction. Moreover, CT-1 positively and negatively correlated with LV mass and ejection fraction, respectively, and the authors concluded that CT-1 was a more sensitive biomarker than the amino-terminal pro-BNP for the identification of heart failure. This latter revelation supports the concept that biomarkers reflective of ongoing ECM activities occurring secondary to pressure overload may be of more value than biomarkers such as BNP that surface and peak only after maladaptive LV myocardial remodeling has occurred. Although this study pertained to pressure overload secondary to hypertension, it seems reasonable to assume that similar results would be obtained if the study population included patients with pressure overload secondary to AS; however, this remains to be proven.

\section{COLLAGEN-DERIVED PEPTIDES}

The myocardial ECM contains collagen types I and III. ${ }^{38}$ After the synthesis of procollagen types I and III within myocardial fibroblasts, these large peptides are released into the extracellular compartment with intact amino- and carboxy-propeptide terminals. Subsequent cleavage by specific matrix proteases generates collagen types I and III fibers and their respective propeptide fragments in a stoichiometric ratio of 1:1. Specifically, the carboxy-terminal and amino-terminal propeptides of procollagen types I and III, respectively, are cleaved and released into the serum yielding collagen types I and III. Because carboxy-terminal and amino-terminal propeptides of procollagen types I and III values, respectively, can be measured in the serum, their values can be used as a surrogate to reflect the overall degree of collagen synthesis. Likewise, collagen type I fibers are degraded by collagenase in a stoichiometric ratio of 1:1. Of note, the small telopeptides (for example, collagen I telopeptide) resulting from the proteolytic processing of collagen type I can also be measured within the serum, and the values can be used to assess the degree of collagen degradation.

Past studies demonstrate that accumulation of myocardial collagen accompanies chronic pressure overload states $^{6,39-41}$ and that increased collagen accumulation can result in increased myocardial stiffness. ${ }^{42}$ Because collagen-derived peptides enter the circulation in quantities proportional to the amount of collagen synthesis and degradation that is simultaneously occurring, ${ }^{38}$ López and colleagues ${ }^{43}$ surmised that measurement of these serum peptides might be used as a means by which to assess the severity and progression of pressure overload states. This supposition was based on past studies that assessed and noted differential serum concentrations of carboxyterminal propeptide (synthesis) and telopeptide (degradation) fragments of procollagen type $I$ in normal and hypertensive patients ${ }^{44}$ and rats. ${ }^{45}$ Specifically, carboxyterminal propeptide was increased in the serum of hypertensive patients ${ }^{44}$ and spontaneously hypertensive rats, ${ }^{45}$ whereas serum telopeptide remained unchanged, thus reflecting a shift in the collagen synthesis/degradation ratio favoring collagen accumulation. Because carboxyterminal propeptide is not myocardial-specific, these investigators assessed the amount of this serum biomarker from both coronary sinus and peripheral blood of hypertensive patients to establish marker specificity for myocardial collagen accumulation. ${ }^{17}$ Values for carboxy-terminal propeptide were higher in coronary sinus serum of hypertensive patients compared with peripheral serum but were not in healthy patients, indicating that measurement of collagen-derived serum peptides may be used as a noninvasive way to assess and follow myocardial fibrosis secondary to pressure overload. Heymans and colleagues ${ }^{41}$ assessed cardiac mRNA expression for collagen types I and III and for collagen synthesis transcripts in patients with chronic pressure overload secondary to AS. Collagen $\alpha 1$ types I and III mRNA levels were significantly higher in patients with AS compared with control patients, as were levels for lysine and proline hydroxylases and procollagen type III $\mathrm{N}$-endopeptidase. It is not surprising that these values were associated with significant increases in total myocardial collagen, with the greatest increase observed at the level of the endocardium. Taken together, the results of these investigations suggest that a role may exist for the serum determination of collagen-derived peptides as a means by which to assess disease severity in the setting of chronic pressure overload.

\section{MATRIX METALLOPROTEINASES AND TISSUE INHIBITORS OF MATRIX METALLOPROTEINASES}

The MMPs are an endogenous family of zinc-dependent enzymes that are intimately associated ECM remodeling processes in numerous disease states, including pressure 
overload. MMPs are categorized into subgroups depending on their substrate specificities. For example, the collagenases include MMP- 1 and 8 and the gelatinases include MMP-2 and $9 .{ }^{46}$ Increases in the plasma levels of MMPs from this latter subgroup have recently been used to predict LV diastolic dysfunction in patients with hypertension ${ }^{16}$ and are particularly pertinent species with respect to this pathology. Although MMPs carry out numerous functions, none is more important than the enzymatic degradation of collagen and other components of the ECM initiated by this family of enzymes.

Under normal circumstances, the TIMPs appropriately counter MMP activity and maintain basal, nonpathologic turnover of the ECM. However, past clinical and experimental studies demonstrate that chronic pressure overload alters this delicate balance and may be partially responsible for the deleterious myocardial changes associated with this disease state. ${ }^{22,24,41,47-50}$ Of the 4 TIMP proteins known to exist, TIMP-4 is remarkable in that it is highly expressed within the myocardium. ${ }^{51}$ TIMP-4 protein levels have been shown to be increased in the plasma and myocardium of patients with LV pressure overload. ${ }^{18,52}$ Although TIMPs bind tightly to and inhibit the majority of the MMP proteins, differences in their inhibitory profiles do exist. For instance, TIMP-1 best inhibits MMP-9 and TIMP-4 best inhibits MMP-2. ${ }^{53}$ These differences suggest that modulation of specific TIMP proteins may have therapeutic potential in modulating LV remodeling secondary to chronic pressure overload.

Past efforts aimed at more clearly defining the MMP:TIMP balance in the context of LV pressure overload have demonstrated that some degree of plasticity exists. For example, Walther and associates ${ }^{24}$ applied supracoronary bands to sheep to recapitulate compensated LVH in an effort to assess ECM gene expression. This technique resulted in a near doubling of the LV mass index and was associated with significant increases in MMP-1, 2, 3, and 9 and TIMP- 1 and 2 gene expression relative to baseline values. The supracoronary bands, which generated a maximum pressure gradient of $56.8 \pm 4.7 \mathrm{~mm} \mathrm{Hg}$, were subsequently removed to reverse the pressure overload state, and complete regression of gene expression levels and myocyte hypertrophy was observed. ${ }^{24}$ Complete reversibility of the latter parameter contrasts the persistent, albeit subtle, cellular changes observed in the clinical investigations carried out by researchers at the University Hospital in Zurich, Switzerland, ${ }^{6-8}$ as described above. Perhaps this discrepancy is secondary to the fact that ovine aortic banding achieved a relatively moderate degree of pressure overload in comparison with those patients with severe and long-standing AS included in past clinical investigations. Nevertheless, Walther and colleagues' animal investigation proved to be instrumental in demonstrating that alterations in gene expression occur within the ECM during induction of LVH and after resolution of the pressure overload state.

The importance of MMP/TIMP balance in the progression of chronic pressure overload has also been investigated in clinical settings. ${ }^{18,41,47,50}$ Although the majority of these clinical studies focused on patients with hypertensive heart disease, ${ }^{41,47,50}$ Fielitz and associates ${ }^{18}$ assessed the regulation of MMPs and TIMPs in patients with severe and longstanding AS. These investigators obtained myocardial biopsies from patients undergoing AVR for AS and quantified mRNA expression for various MMPs and TIMPs, as well as MMP and TIMP protein levels. mRNA expression and protein levels for MMP-2 and extracellular matrix metalloproteinase inducer were significantly greater than those values obtained from the control hearts, whereas MMP-9 and TIMP-1 mRNA and protein levels were decreased. Although TIMP-4 mRNA expression was not increased relative to control values, TIMP-4 protein levels were, perhaps as a result of some degree of post-transcriptional modulation. With respect to the MMP/TIMP protein level ratio in the setting of severe AS, the overall balance appeared shifted toward MMP inhibition, thereby favoring collagen accumulation.

\section{PHARMACOLOGIC STRATEGIES, CLINICAL IMPLICATIONS, AND FUTURE DIRECTIONS}

Recent clinical investigations suggest that pharmacologic intervention can influence the degree of observed LV mass regression in patients with LV pressure overload. For instance, Devereux and colleagues ${ }^{54}$ demonstrated that modulation of the renin-angiotensin system using angiotensin receptor blockade (ARB) achieved greater LV mass regression than administration of conventional beta-blockade therapy in patients with arterial hypertension and LVH. On the basis of these data, it would seem likely that a similar pharmacologic strategy could be used to achieve like results in the postoperative management of patient undergoing AVR for AS. Indeed, Dahl and colleagues ${ }^{55}$ observed just such a beneficial result in their single-center prospective randomized and blinded investigation. Specifically, these investigators applied ARB to patients undergoing AVR for AS and compared their postoperative LV mass indices against those patients receiving conventional medical therapy after AVR for AS. One year later, LV mass index was significantly lower in the ARB group. It is noteworthy that despite these results, there is at present no uniformity with respect to recommended pharmacologic interventions post-AVR. Moreover, no strategies exist that specifically target adverse matrix remodeling that occurs with AS and persists after AVR. Thus, combining potential biomarkers of matrix remodeling with more standardized pharmacologic protocols may provide an important future direction. 


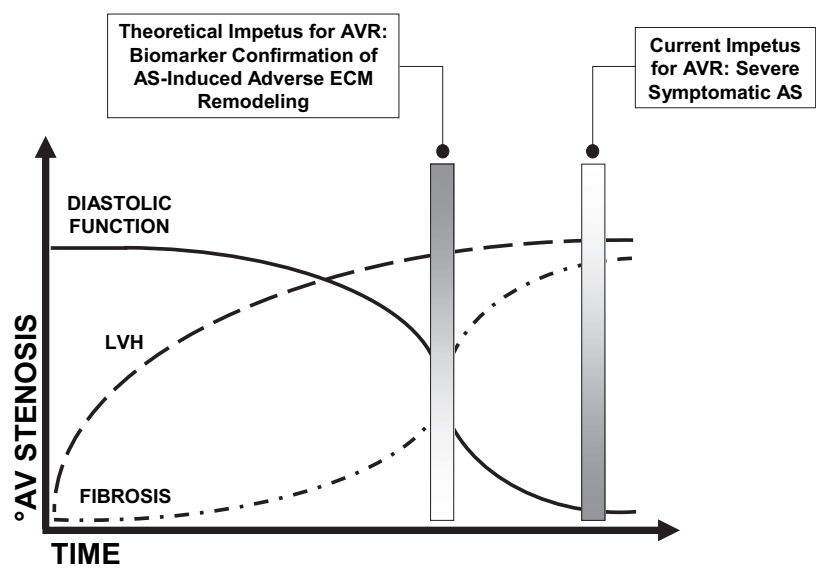

FIGURE 3. The hypothetic schematic depicts deterioration in diastolic function and an increase in myocyte hypertrophy and myocardial fibrosis over time and in the setting of AS-induced progressive pressure overload. Current management paradigms advocate AVR for patients with severe AS and associated symptoms, namely, angina, syncope, and chronic heart failure. However, AVR carried out only after symptom onset likely fails to reverse the maladaptive ECM remodeling that has already occurred. Identification of a biomarker capable of heralding the transition from AS-induced adaptive to maladaptive ECM remodeling processes may have therapeutic value. Specifically, identification of such a biomarker may be used to direct more timely elimination of the pressure overload state, thus optimizing the chances for normalization within the ECM and improved postoperative outcomes. $A S$, Aortic stenosis; $A V$, aortic valve; $A V R$, aortic valve replacement; $E C M$, extracellular matrix; $L V H$, left ventricular hypertrophy.

\section{CONCLUSIONS}

Although forestalling AVR until the onset of symptoms has proven to be a relatively successful strategy for patients with severe AS for many years, it is apparent that intervention delayed until this time point fails to achieve complete regression of maladaptive changes that occur within the myocardial ECM. Persistence of the products of myocardial ECM elaboration secondary to AS may translate into a postoperative clinical condition characterized by a continued increase in myocardial stiffness, that is, diastolic dysfunction. The summation of remodeling events as they relate to maladaptive phases of AS and $\mathrm{LVH}$, and their temporal relation to the development of heart failure and AVR, are shown in Figure 3. As depicted by the hypothetic figure, cellular and ECM remodeling occurs during the progression of AS. In addition, clinical studies suggest that myocardial fibrosis persists after AVR for AS and highlights potential inadequacies of current treatment guidelines. Identification of a matrix biomarker that reflects the transition to maladaptive remodeling in the setting of LV pressure overload may provide an opportunity to achieve more complete regression of deleterious changes within the ECM at the time of AVR. Targeting the ECM, and identification of just such a biomarker, may ultimately provide clinicians with a more precise means by which they are able to assess and counsel their patients with severe, though perhaps asymptomatic, AS.

\section{References}

1. Grossman W, Jones D, McLaurin LP. Wall stress and patterns of hypertrophy in the human left ventricle. J Clin Invest. 1975;53:332-41.

2. Badeer HS. Biological significance of cardiac hypertrophy. Am J Cardiol. 1964; 14:133-8.

3. Alpert NR. Preface. Cardiac hypertrophy. New York, NY: Academic Press, Inc; 1971:XV-XVI.

4. Carabello BA, Paulus WJ. Aortic stenosis. Lancet. 2009;373:956-66.

5. Monrad ES, Hess OM, Murakami T, et al. Time course of regression of left ventricular hypertrophy after aortic valve replacement. Circulation. 1988;77: 1345-55.

6. Villari B, Vassalli G, Monrad ES, et al. Normalization of diastolic dysfunction in aortic stenosis late after valve replacement. Circulation. 1995;91:2353-8.

7. Krayenbuehl HP, Hess OM, Monrad ES, et al. Left ventricular myocardial structure in aortic valve disease before, intermediate, and late after aortic valve replacement. Circulation. 1989;79:744-55.

8. Hess OM, Ritter M, Schneider J, et al. Diastolic stiffness and myocardial structure in aortic valve disease before and after valve replacement. Circulation. 1984; 69:855-65.

9. Peterson KL, Tsuji J, Johnson A, et al. Diastolic left ventricular pressure-volume and stress-strain relations in patients with valvular aortic stenosis and left ventricular hypertrophy. Circulation. 1978;58:77-89.

10. Hein S, Arnon E, Kostin S, et al. Progression from compensated hypertrophy to failure in the pressure-overloaded human heart: structural deterioration and compensatory mechanisms. Circulation. 2003;107:984-91.

11. Bonow RO, Carabello BA, Chatterjee K, et al. 2008 focused update incorporated into the ACC/AHA 2006 guidelines for the management of patients with valvular heart disease: a report of the American College of Cardiology/American Heart Association Task Force on Practice Guidelines (Writing Committee to revise the 1998 guidelines for the management of patients with valvular heart disease). Endorsed by the Society of Cardiovascular Anesthesiologists, Society for Cardiovascular Angiography and Interventions, and Society of Thoracic Surgeons. J Am Coll Cardiol. 2008;23:e1-142.

12. Ross J Jr, Braunwald E. Aortic stenosis. Circulation. 1968;38:61-7.

13. Azevedo CF, Nigri M, Higuchi ML, et al. Prognostic significance of myocardial fibrosis quantification by histopathology and magnetic resonance imaging in patients with severe aortic valve disease. J Am Coll Cardiol. 2010;56: 278-87.

14. Monrad ES, Hess OM, Murakami T, et al. Abnormal exercise hemodynamics in patients with normal systolic function late after aortic valve replacement. Circulation. 1988;77:613-24.

15. Weidemann F, Herrmann S, Störk S, et al. Impact of myocardial fibrosis in patients with symptomatic severe aortic stenosis. Circulation. 2009;120:577-84.

16. Martos R, Baugh J, Ledwidge M, et al. Diastolic heart failure: evidence of increased myocardial collagen turnover linked to diastolic dysfunction. Circulation. 2007;115:888-95.

17. Querejeta R, López B, González A, et al. Increased collagen type I synthesis in patients with heart failure of hypertensive origin: relation to myocardial fibrosis. Circulation. 2004;110:1263-8.

18. Fielitz J, Leuschner M, Zurbrügg HR, et al. Regulation of matrix metalloproteinases and their inhibitors in the left ventricular myocardium of patients with aortic stenosis. J Mol Med. 2004;82:809-20.

19. Fielitz J, Hein S, Mitrovic V, et al. Activation of the cardiac renin-angiotensin system and increased myocardial collagen expression in human aortic valve disease. J Am Coll Cardiol. 2001;37:1443-9.

20. Schwarz F, Flameng W, Schaper J, et al. Correlation between myocardial structure and diastolic properties of the heart in chronic aortic valve disease: effects of corrective surgery. Am J Cardiol. 1978;42:895-903.

21. Moorjani N, Ahmad M, Catarino P, et al. Activation of apoptotic caspase cascade during the transition to pressure overload-induced heart failure. J Am Coll Cardiol. 2006;48:1451-8.

22. Schubert A, Walther T, Falk V, et al. Extracellular matrix gene expression correlates to left ventricular mass index after surgical induction of left ventricular hypertrophy. Basic Res Cardiol. 2001;96:381-7.

23. Nagatomo Y, Carabello BA, Coker ML, et al. Differential effects of pressure or volume overload on myocardial MMP levels and inhibitory control. Am J Physiol Heart Circ Physiol. 2000;278:H151-61. 
24. Walther T, Schubert A, Falk V, et al. Regression of left ventricular hypertrophy after surgical therapy for aortic stenosis is associated with changes in extracellular matrix gene expression. Circulation. 2001;104(suppl I):I-54-8.

25. Gerber IL, Stewart RA, Legget ME, et al. Increased plasma natriuretic peptide levels reflect symptom onset in aortic stenosis. Circulation. 2003;107:1884-90.

26. Imai $\mathrm{K}$, Okura $\mathrm{H}$, Kume $\mathrm{T}$, et al. C-reactive protein predicts severity, progression, and prognosis of asymptomatic aortic valve stenosis. Am Heart J. 2008;156:713-8.

27. Galante A, Pietrouiusti A, Vellini M, et al. C-reactive protein is increased in patients with degenerative aortic valvular stenosis. J Am Coll Cardiol. 2001;38: 1078-82.

28. Ferrari G, Sainger R, Beckmann E, et al. Validation of plasma biomarkers in degenerative calcific aortic stenosis. J Surg Res. 2010;163:12-7.

29. Neverdal NO, Knudsen CW, Husebye T, et al. The effect of aortic valve replacement on plasma B-type natriuretic peptide in patients with severe aortic stenosisone year follow-up. Eur J Heart Fail. 2006;8:257-62.

30. Blobe GC, Schiemann WP, Lodish HF. Role of transforming growth factor beta in human disease. $N$ Engl J Med. 2000;342:1350-8.

31. Petrov VV, Fagard RH, Lijnen PJ. Stimulation of collagen production by transforming growth factor-betal during differentiation of cardiac fibroblasts to myofibroblasts. Hypertension. 2002;39:258-63.

32. Villar AV, Llano M, Cobo M, et al. Gender differences of echocardiographic and gene expression patterns in human pressure overload left ventricular hypertrophy. J Mol Cell Cardiol. 2009;46:526-35.

33. Kuwahara F, Kai H, Tokuda K, et al. Transforming growth factor-beta function blocking prevents myocardial fibrosis and diastolic dysfunction in pressureoverloaded rats. Circulation. 2002;106:130-5.

34. Kuwahara K, Saito Y, Harada M, et al. Involvement of cardiotrophin-1 in cardiac myocyte-nonmyocyte interactions during hypertrophy of rat cardiac myocytes in vitro. Circulation. 1999; 100:1116-24.

35. Pennica D, King KL, Shaw KJ, et al. Expression cloning of cardiotrophin 1, a cytokine that induces cardiac myocyte hypertrophy. Proc Natl Acad Sci U S A. 1995;92:1142-6.

36. Tsuruda T, Jougasaki M, Boerrigter G, et al. Cardiotrophin-1 stimulation of cardiac fibroblast growth: roles for glycoprotein 130/leukemia inhibitory factor receptor and the endothelin type A receptor. Circ Res. 2002;90:128-34.

37. López B, González A, Querejeta R, et al. Association of plasma cardiotrophin-1 with stage $\mathrm{C}$ heart failure in hypertensive patients: potential diagnostic implications. J Hypertens. 2009;27:418-24.

38. Nimmi ME. In: Zern MA, Reid LM, eds. Fibrillar collagens: their biosynthesis, molecular structure, and mode of assembly. Extracellular Matrix. New York: Marcel Decker; 1993. p. 121-48.

39. Tanaka M, Fujiwara H, Onodera T, et al. Quantitative analysis of myocardial fibrosis in normal, hypertensive hearts, and hypertrophic cardiomyopathy. Br Heart J. 1986;55:575-81.

40. Rossi MA. Pathologic fibrosis and connective tissue matrix in left ventricular hypertrophy due to chronic arterial hypertension in humans. J Hypertens. 1998;16: $1031-41$.

41. Heymans S, Schroen B, Vermeersch P, et al. Increased cardiac expression of tissue inhibitor of metalloproteinase- 1 and tissue inhibitor of metalloproteinase- 2 is related to cardiac fibrosis and dysfunction in the chronic pressure-overloaded human heart. Circulation. 2005;112:1136-44.
42. Díez J, Querejeta R, López B, et al. Losartan-dependent regression of myocardial fibrosis is associated with reduction of left-ventricular chamber stiffness in hypertensive patients. Circulation. 2002;105:2512-7.

43. López B, González A, Querejeta R, et al. The use of collagen-derived serum peptides for the clinical assessment of hypertensive heart disease. J Hypertens. 2005; 23:1445-51.

44. Díez J, Laviades C, Mayor G, et al. Increased serum concentrations of procollagen peptides in essential hypertension. Relation to cardiac alterations. Circulation. 1995;91:1450-6.

45. Díez J, Panizo A, Gil MJ, et al. Serum markers of collagen type I metabolism in spontaneously hypertensive rats: relation to myocardial fibrosis. Circulation. 1996;93:1026-32.

46. Deardorff R, Spinale FG. Cytokines and matrix metalloproteinases as potential biomarkers in chronic heart failure. Biomark Med. 2009;3:513-23.

47. Ahmed SH, Clark LL, Pennington WR, et al. Matrix metalloproteinases/tissue inhibitors of metalloproteinases: relationship between changes in proteolytic determinants of matrix composition and structural, functional, and clinical manifestations of hypertensive heart disease. Circulation. 2006;113:2089-96.

48. Foronjy RF, Sun J, Lemaitre V, et al. Transgenic expression of matrix metalloproteinase-1 inhibits myocardial fibrosis and prevents the transition to heart failure in a pressure overload mouse model. Hypertens Res. 2008;31:725-35.

49. Matsusaka H, Ide T, Matsushima S, et al. Targeted deletion of matrix metalloproteinase 2 ameliorates myocardial remodeling in mice with chronic pressure overload. Hypertension. 2006;47:711-7.

50. González A, López B, Querejeta R, et al. Filling pressures and collagen metabolism in hypertensive patients with heart failure and normal ejection fraction. $\mathrm{Hy}$ pertension. 2010;55:1418-24.

51. Melendez-Zajgla J, Del Pozo L, Ceballos G, et al. Tissue inhibitor of metalloproteinases-4. The road less traveled. Mol Cancer. 2008;21(7):85.

52. Stroud RE, Deschamps AM, Lowry AS, et al. Plasma monitoring of the myocardial specific tissue inhibitor of metalloproteinase-4 after alcohol septal ablation in hypertrophic obstructive cardiomyopathy. J Card Fail. 2005;11:124-30.

53. Cauwe B, Van den Steen PE, Opdenakker G. The biochemical, biological, and pathological kaleidoscope of cell surface substrates processed by matrix metalloproteinases. Crit Rev Biochem Mol Biol. 2007;42:113-85.

54. Devereux RB, Dahlöf B, Gerdts E, et al. Regression of hypertensive left ventricular hypertrophy by losartan compared with atenolol: the Losartan Intervention for Endpoint Reduction in Hypertension (LIFE) trial. Circulation. 2004;110: 1456-62.

55. Dahl JS, Videbaek L, Poulsen MK, et al. Effect of candesartan treatment on left ventricular remodeling after aortic valve replacement for aortic stenosis. Am J Cardiol. 2010;106:713-9.

56. Yu PJ, Skolnick A, Ferrari G, et al. Correlation between plasma osteopontin levels and aortic valve calcification: potential insights into the pathogenesis of aortic valve calcification and stenosis. J Thorac Cardiovasc Surg. 2009;138:196-9.

57. Van Lint P, Libert C. Chemokine and cytokine processing by matrix metalloproteinases and its effect on leukocyte migration and inflammation. J Leukoc Biol. 2007;82:1375-81.

58. Frantz S, Störk S, Michels K, et al. Tissue inhibitor of metalloproteinases levels in patients with chronic heart failure: an independent predictor of mortality. Eur J Heart Fail. 2008;10:388-95. 